\title{
Alterstice
}

Revue internationale de la recherche interculturelle

International Journal of Intercultural Research

Revista International de la Investigacion Intercultural

\section{Pour une approche interculturelle en travail social. Théories et pratiques, de $\mathrm{M}$. Cohen-Emerique}

\section{Alexandra Boilard et Yvan Leanza}

Volume 1, numéro 1, 2011

URI : https://id.erudit.org/iderudit/1077595ar

DOI : https://doi.org/10.7202/1077595ar

Aller au sommaire du numéro

Éditeur(s)

Alterstice

ISSN

1923-919X (numérique)

Découvrir la revue

Citer ce compte rendu

Boilard, A. \& Leanza, Y. (2011). Compte rendu de [Pour une approche

interculturelle en travail social. Théories et pratiques, de M. Cohen-Emerique].

Alterstice, 1(1), 101-105. https://doi.org/10.7202/1077595ar d'utilisation que vous pouvez consulter en ligne.

https://apropos.erudit.org/fr/usagers/politique-dutilisation/ 


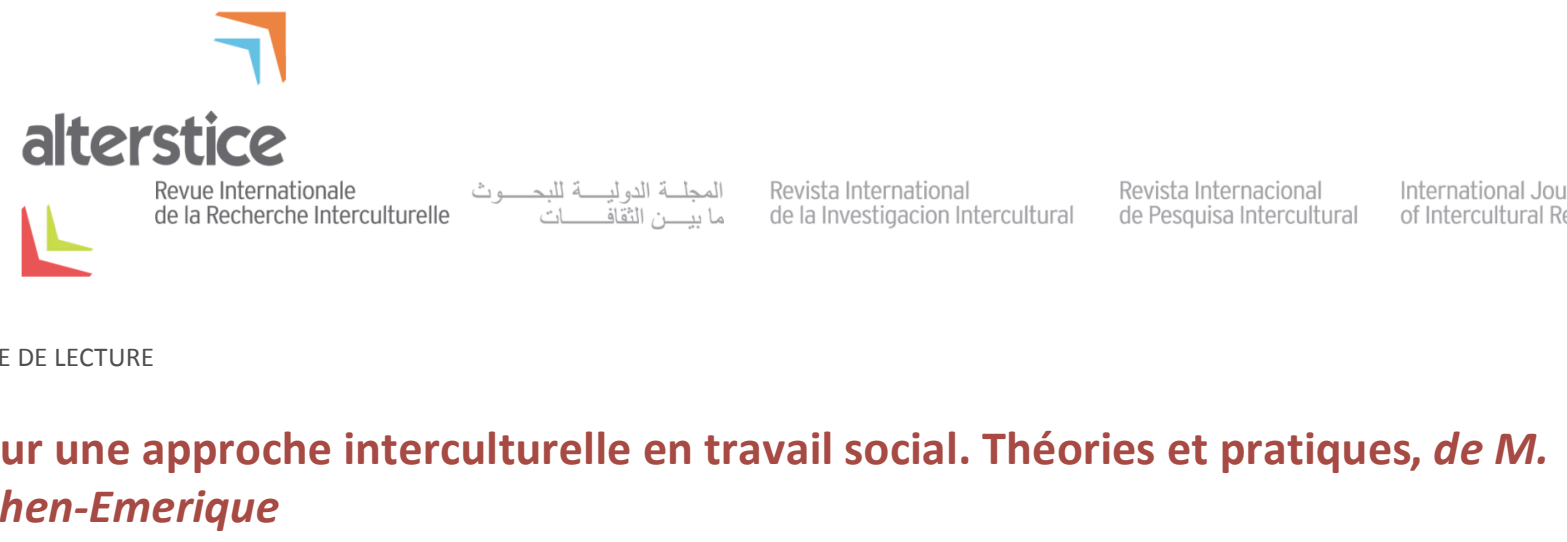

Alexandra Boilard ${ }^{1}$ et Yvan Leanza ${ }^{1}$

\section{Rattachement des auteurs \\ ${ }^{1}$ Laboratoire Psychologie et Cultures, École de Psychologie, Université Laval, Québec, Canada}

\section{Correspondance}

alexandra.boilard.1@ulaval.ca

\section{Références de l'ouvrage :}

Cohen-Emerique, M. (2011). Pour une approche interculturelle en travail social. Théories et pratiques. France: Presses de I'EHESP. (ISBN : 978-2-8109-0053-4)

\section{Pour citer cet article :}

Boilard, A. et Leanza, Y. (2011). Pour une approche interculturelle en travail social. Théories et pratiques [Note de lecture]. Alterstice, 1(1), 101-106.

Le livre intitulé Pour une approche interculturelle en travail social. Théories et pratiques est un ouvrage de 435 pages (deux parties, six sections et 20 chapitres) qui fait la synthèse des travaux de recherche, réflexions et propositions pratiques de l'auteure. Margalit Cohen-Emerique est psychosociologue, chercheuse et formatrice, mais elle a aussi expérimenté les migrations. Elle a amorcé sa carrière professionnelle en tant que psychologue clinicienne au Ministère de la Santé à Jérusalem. Collaboratrice de Carmel Camilleri, elle a enseigné à l'Université de Montréal, à l'Université catholique de Milan et à l'École supérieure de travail social (ETSUP) de Paris. Elle a écrit de nombreux articles et participé à de nombreuses publications traitant de "l'interculturel ».

L'objet premier du livre est l'expérience de la différence culturelle dans le travail social. S’appuyant sur sa grande expérience en recherche comme en formation, l'auteure explique les obstacles les plus couramment vécus par les travailleurs sociaux, en apportant des solutions concrètes, tout en retraçant son cheminement intellectuel. 


\title{
Première partie : L'intervention sociale auprès des migrants et de leurs familles : du constat de ses difficultés à l’approche interculturelle
}

\author{
Section 1 : Quelle formation pour les travailleurs sociaux? Délimitation du champ de recherche et des \\ objectifs de formation
}

Les travailleurs sociaux sont confrontés dans leur pratique à plusieurs types de problèmes qui fragilisent les personnes vivant un processus migratoire. Les professionnels doivent ainsi s'adapter aux multiples demandes provenant de cette clientèle, mais également aux multiples changements possibles en fonction de la diversité des pays d'origine et des événements qui sont survenus pendant le processus migratoire. Or, les travailleurs sociaux n'ont pas les repères adéquats pour effectuer les évaluations qu'ils doivent réaliser et qui sont la quintessence du travail social. C'est dans ce contexte que Cohen-Emerique se penche sur la question de leur formation spécifique en vue d'ajuster leurs pratiques au contexte interculturel. Un constat émerge au chapitre 2 : les formations existantes sur la diversité réduisent la culture à quelques traits folkloriques peu utiles à l'expertise professionnelle, mais le manque de recherches et l'idéologie politique dominante en France, qui exclut la diversité culturelle et la migration comme élément différenciateur dans la sphère publique (et donc dans les interventions des institutions), ont aussi leur part de responsabilité dans les défaillances de la formation, selon l'auteure.

Traversant différents champs théoriques et de nombreuses disciplines, Cohen-Emerique décrit dans le chapitre 3 les concepts nécessaires à la construction d'une compréhension en nuance du rapport à l'Autre. Elle évoque ainsi l'acculturation, la communication interculturelle, les représentations sociales et bien d'autres, pour aboutir à la méthode du choc culturel. Ce choc joue un rôle fondamental dans la relation entre travailleurs sociaux et migrants. Il est un révélateur des représentations, des normes, des valeurs et attitudes personnelles à partir desquelles il est possible de donner un sens aux situations vécues et permet d'orienter, si l'on s'y attarde, les interventions.

Section 2 : Surmonter les obstacles à la tolérance et au respect de l'autre pour élaborer l'approche interculturelle

L'ensemble de cette section est un peu à l'image du dernier chapitre de la section précédente: il s'agit essentiellement d'une (longue) liste explicative de concepts empruntés à diverses disciplines et nécessaires à une théorisation des relations interculturelles dans l'intervention sociale. Dans un premier temps (chapitre 4), l'auteure traite des concepts de migrant, culture, identité et intégration. Elle montre que chacun de ces concepts nécessite une définition et un usage nuancés. Elle revient par exemple sur la représentation globalisante et figée de la culture, dont il faudrait se défaire. Au contraire, la culture, soutient-elle, est en constante évolution et peut varier selon les différentes perspectives étudiées. Le professionnel se doit d'éviter les généralisations et se doit également d'amener le migrant à rendre compte de son propre cadre de référence par rapport à ses différentes appartenances.

Ensuite (chapitre 5), Cohen-Emerique traite des obstacles à la communication et à la compréhension. Ces mécanismes fortement ancrés chez les individus interagissent dans les relations entre professionnels et migrants. Par exemple, les préjugés et les stéréotypes sont considérés comme des obstacles de taille à la communication interculturelle. Presque mécaniquement, certains professionnels les utiliseront pour tenter d'expliquer des situations difficiles à comprendre et par là même seront amenés à commettre des erreurs d'évaluation ou d'intervention.

Deux attitudes de valorisation de l'Autre, bien qu'elles puissent sembler positives au premier regard, entravent en réalité la relation: l'exotisme et la minimisation. Elles sont utilisées par les professionnels, ce que présente le chapitre 6, qui traite plus généralement des ethnocentrismes. L'exotisme est défini comme étant un rêve de dépaysement, une approche idéalisée de la culture de l'Autre. Quant à la minimisation, elle est le désir absolu de se rapprocher de l'Autre quelle que soit son origine. Même si ces attitudes peuvent sembler positives aux yeux du professionnel, elles se traduisent négativement aux yeux de la personne migrante.

Ces mécanismes sont le produit de l'ethnocentrisme propre à tout groupe, mais aussi à un aspect spécifique de la socialisation professionnelle des travailleurs sociaux: le modèle individualiste du sujet (chapitre 7). C'est, selon 
l'auteure, une conception de la personne qui fait écran à la compréhension de situation impliquant des migrants de sociétés non occidentales.

Pour pallier ces nombreuses difficultés, Cohen-Emerique a développé une approche qu'elle nomme interculturelle. Dans le chapitre 8, reproduit en presque totalité dans ce numéro d'Alterstice, c'est la notion même d'interculturel qui est discutée. Le chapitre 9 présente les grandes lignes de l'approche interculturelle. L'auteure choisit le terme approche plutôt que compétence pour indiquer qu'il s'agit d'un processus laissant place au contexte et à la complexité, plutôt qu'à une "recette comportementale ». L'approche permet en premier lieu de cerner le professionnel dans sa globalité et comme élaborateur de sens, en interaction constante avec l'Autre. L'Autre étant vu comme un élaborateur de sens tout aussi important. Elle permet également de se décentrer, en réfléchissant sur soi, et de faire émerger à la conscience son propre cadre de référence. En second lieu, cette approche permet de découvrir le cadre de référence du migrant, sans en accepter nécessairement les prémisses et les fins. Il s’agit de s'intéresser aux différences culturelles, mais au-delà de celles-ci, il s'agit de porter attention aux identités liées au processus migratoire, à l'exil et à tous les traumatismes qui peuvent y être liés. Cette approche est présentée en détail dans la seconde partie du livre.

\section{Deuxième partie : Les trois démarches de l’approche interculturelle : la décentration, la découverte du cadre de référence de l’autre, la négociation et la médiation interculturelles.}

\section{Section 1 : La décentration}

L'auteure explique que la décentration vise deux objectifs. Le premier est de cerner le cadre de référence à partir duquel le professionnel perçoit l'Autre au moment du choc culturel. Le second étant de repérer et d'analyser les "zones sensibles " à propos desquelles le professionnel a du mal à communiquer avec le migrant. Certains thèmes, considérés comme des "zones sensibles », mettent souvent mal à l’aise les professionnels : la sociabilité (les codes de bienséance, les cadeaux...) mais aussi la mise en scène du corps (habillement, odeur...), la perception différentielle du temps, les pratiques religieuses, les rapports hommes-femmes, l'éducation des enfants et la scolarisation. Les chapitres 11 à 14 traitent de ces divers thèmes sur mode à la fois descriptif (peut-être en contradiction avec la volonté exprimée précédemment de ne pas enfermer l'Autre dans une culture définie une fois pour toutes) et sensible dans la mesure où de nombreuses situations vécues sont présentées et analysées.

Le dernier chapitre de cette section (15) met en évidence les concepts clés et les méthodes qui font obstacle à la décentration. Ils sont enseignés dans les écoles de travail social, mais aussi entretenus par la pression des pairs ou par la hiérarchie dans les institutions. Par exemple, la non-implication de l'aidant dans la relation d'aide serait le garant du professionnalisme. Or, comme le démontre Cohen-Emerique, cette non-implication peut être perçue par le client comme un manque d'intérêt voire comme une attitude méprisante. II s'agit alors de savoir (et pouvoir) s'impliquer de façon consciente et contrôlée à des moments choisis par le professionnel. En définitive, l'intervention sociale en milieu pluriculturel est complexe et nécessite souplesse et relativisme.

\section{Section 2 : À la découverte du cadre de référence de l'autre}

La décentration est nécessaire dans un contexte de relation interculturelle, toutefois ce processus doit être complémentaire à celui de découvrir les cadres de référence de l'Autre. En général, les professionnels éprouvent de la difficulté à le faire pour des personnes migrantes. Ces difficultés sont détaillées dans les chapitres 16 et 17 , le premier étant centré sur les difficultés contextuelles et communicationnelles, le second sur la prépondérance de la grille de lecture psychologique pour expliquer les comportements des migrants (en écho avec le modèle individualiste du sujet présenté au chapitre 7). Une des difficultés récurrentes vécues par les professionnels est la barrière de la langue. Des traducteurs professionnels seraient nécessaires pour faire passer le contenu des messages, or ce sont en général les personnes accompagnant le client qui exercent la traduction. Malgré le désir de productivité dans les institutions, les professionnels doivent prendre le temps d'analyser les éléments contextuels pour maximiser la compréhension et faciliter l'exploration du cadre de référence.

Pour contrer cette difficulté, la proposition de Cohen-Emerique est d'apporter aux professionnels des connaissances supplémentaires sur les processus d'acculturation et de construction identitaire (chapitre 18). 
Cependant, l'auteur reconnaît que les positions des théoriciens en la matière sont parfois antinomiques et peuvent semer le doute chez les intervenants qui reçoivent des injonctions contradictoires. Elle mentionne l'existence d'au moins deux courants qui s'ostracisent mutuellement : les tenants d'une approche socio-économique et les tenants d'une approche qui attache une importance majeure aux facteurs culturels. Les premiers reprochent aux seconds de réifier la culture, les seconds accusent les premiers de l'oublier un peu vite. Cohen-Emerique propose d'intégrer les deux afin d'aider à comprendre les processus migratoires. Dans la formation des professionnels, une attention particulière devrait être portée au rôle que le migrant prend dans ses choix acculturatifs et à la reconnaissance des différentes formes d'intégration possible. In fine, l'objectif pour les travailleurs sociaux serait d'accompagner les migrants dans leurs processus identitaires acculturatifs, dans une identification positive aux deux cultures, en reconnaissant l’histoire personnelle et en les laissant prendre leurs propres décisions.

\section{Section 3 : La négociation et la médiation interculturelles}

Cette section expose les deux processus par lesquels il est possible de construire des solutions aux conflits de valeurs qui surgissent dans l'intervention sociale: la négociation interculturelle (chapitre 19) et la médiation interculturelle (chapitre 20). La négociation est le processus par lequel on engage une série d'échanges où chacun respectera autrui dans son intégrité et ses valeurs tout en aboutissant à un compromis qui fait sens pour tous. Une médiation, quant à elle, permet de mettre d'accord, de concilier ou réconcilier des personnes ou encore des parties par l'intervention d'un tiers.

Pratiquer la négociation (inter)culturelle implique un investissement et une motivation particulière de la part des professionnels pour résoudre les conflits de valeurs. Plusieurs approches (psychosociologique, sociologique, psychanalytique, ethnologique, émancipation, action sociale) sont présentées. Toutes s'entendent pour dire qu'il n'y a pas qu'une seule réponse (ou une recette) pour gérer les conflits de valeurs. Selon Cohen-Emerique, les quatre préalables à la négociation sont :

1. la reconnaissance qu'il y a conflit de valeur et non un comportement aberrant voire pathologique;

2. la reconnaissance que le client est un partenaire dans la recherche de solutions;

3. une démarche de rapprochement, ce qui est une difficulté majeure pour des intervenants qui attendent toujours un changement de la part des migrants;

4. une double grille de lecture : celle des appartenances culturelles et des ruptures de ces appartenances et celle des stratégies d'adaptation (ou acculturation).

Bien entendu, les intervenants sur le terrain ne peuvent porter seuls cette tâche de négociation. Le contexte institutionnel (la hiérarchie), et plus largement sociopolitique, doit étayer par des décisions claires ce processus pour qu'il aboutisse.

Le chapitre sur la médiation interculturelle montre les différentes méthodes que les médiatrices mettent en œuvre pour établir des passerelles entre travailleurs sociaux et migrants: respect des hiérarchies, des codes de communication phatique... En plus de saisir et faire comprendre les écarts entre ici et là-bas, les médiatrices appliquent une approche systémique qui cherche à protéger l'unité familiale, jouant un rôle de réassurance auprès de la population migrante. Cette approche permet de trouver l'équilibre souhaité entre les pratiques d'origine et le changement désiré pour adoucir les transitions vécues. Toutefois, il demeure important que les médiateurs collaborent avec les travailleurs sociaux, afin qu'ils transmettent leur compréhension des problèmes et permettent d'ouvrir les barrières qui entravent la communication.

\section{Conclusion}

Dans sa conclusion Cohen-Emerique rappel à quel point la communication interculturelle est parsemée d'embûches. Elle enjoint les équipes sur le terrain, les instances représentant les professionnels et les institutions sociales à entamer une réflexion sur la place de l'altérité et à travailler au développement d'«actions interculturelles ", c'est-à-dire d'un ensemble de pratiques collectives et individuelles qui font le pari de la négociation. L'auteure insiste aussi sur diverses modalités de formation, nécessaires pour développer chez les professionnels les ressources nécessaires à l'intervention en contexte multiculturel. La recherche multidisciplinaire serait la voie royale pour établir de nouvelles connaissances et pratiques en la matière.

Alterstice - Revue Internationale de la Recherche Interculturelle, vol. $1, n^{\circ} 1$ 


\section{Commentaires}

Tout au long de l'ouvrage, l'auteure explique les concepts avec grande pédagogie, tout en s'appuyant sur des exemples tirés d'expériences de terrain des professionnels qu'elle a rencontrés, ce qui enrichit la lecture. CohenEmerique ne tente pas seulement de démontrer les incohérences des institutions françaises (aussi bien formatives que d'actions sociales) qui ne prennent pas en considération la diversité. Au contraire, elle offre plusieurs solutions pour former plus adéquatement les travailleurs sociaux et pour améliorer la qualité de leurs interventions (et leur bien-être comme professionnels). Elle explique les faits tels qu'ils sont rencontrés par les professionnels et construit des liens avec des notions théoriques utiles à l'intervention interculturelle. Nous constatons toutefois que, malgré son expérience et ses démonstrations admirables, Cohen-Emerique n'est pas non plus à l'abri des difficultés entraînées par la tension entre la réification culturelle et la souplesse qu'elle défend.

Du point de vue de la forme, on note quelques longueurs, parfois même de longs passages très descriptifs sur des notions théoriques.

C'est un ouvrage qui mène inévitablement vers de nouvelles réflexions et sur un questionnement à propos de notre façon, consciente ou pas, d'être ethnocentrique dans nos pensées, attitudes et actions. II nous semble que la contribution la plus importante de cet ouvrage est une compréhension approfondie de la complexité des interactions avec l'Autre. Espérons que ce livre permette de discuter de cette problématique au sein des écoles de travail social et dans toute autre institution de formation et d'intervention sociosanitaire. 